\title{
Instabilities in dielectric elastomers: buckling, wrinkling, and crumpling
}

Hareesh Godaba' ${ }^{1}$ Zhi-Qian Zhang², Ujjaval Gupta' ${ }^{1}$, Choon Chiang Foo², Jian Zhu',a)

${ }^{1}$ Department of Mechanical Engineering, National University of Singapore, 9 Engineering Drive 1, Singapore 117576

${ }^{2}$ Institute of High Performance Computing, 1 Fusionopolis Way, \#16-16 Connexis, Singapore 138632

\begin{abstract}
Instabilities in a thin sheet are ubiquitous and can be induced by various stimuli, such as a uniaxial force, a liquid-vapor surface tension, etc. This paper investigates voltage-induced instabilities in a membrane of a dielectric elastomer. Instabilities including buckling, wrinkling, and crumpling are observed in the experiments. The prestretches of the dielectric elastomer are found to play a significant role in determining its instability mode. When the prestretch is small, intermediate, or large, the membrane may undergo buckling, wrinkling, or crumpling, respectively. Finite element analysis is conducted to study these instability modes, and the simulations are well consistent with the experimental observations. We wish that this investigation of mechanical and physical properties of dielectric elastomers can enhance their extensive and significant applications to soft devices and soft robots.
\end{abstract}

*Corresponding author: mpezhuj@nus.edu.sg

Hareesh Godaba is currently a postdoctoral research associate at Centre for Advanced Robotics, Queen Mary University of London, Mile End Road, London E1 4NS. 


\section{Introduction}

Instabilities in a thin sheet are ubiquitous and can lead to various morphological patterns. A thin membrane buckles when compressed along its boundaries. Smooth wrinkling and sharp crumpling are familiar motifs in biological or synthetic sheets, such as plant leaves, fish skins, and crushed foils. These instabilities can be induced by various stimuli. Lateral wrinkles may appear on a sheet subject to a uniaxial tensile force ${ }^{1}$. Thin gel sheets that undergo non-uniform shrinkage may exhibit various buckling or wrinkling patterns, depending on the nature of embedding of non-Euclidean metrics ${ }^{2}$. An elastic sheet stretched over a liquid drop may reveal wrinkling and crumpling as distinct symmetry-breaking instabilities ${ }^{3}$.

A dielectric elastomer, consisting of a thin membrane of elastomer sandwiched between two compliant electrodes ${ }^{4-6}$, is susceptible to voltage-induced instabilities. The voltage applied between the two electrodes, can lead to the Maxwell stress, which usually causes the membrane of elastomer to expand in area and shrink in thickness. A membrane of a dielectric elastomer may undergo instability, when the applied voltage reaches a critical level. Different instability modes were observed in the experiments. For example, Rosset et al. studied buckling in a circular membrane of a dielectric elastomer, which is absent of prestretches ${ }^{7}$. The membrane was found to bulge with finite out-of-plane displacements at a certain level of voltage. This voltage-induced buckling has recently been employed to pump fluid within microfluidic channels ${ }^{8}$. More recently, buckling instabilities were also analyzed in freely floating dielectric elastomer sheets under voltage actuation 9. On the other hand, another circular membrane, subject to equal-biaxial prestretches, was found to form wrinkles locally with the flat and wrinkled regions coexistent on its surfaces, with negligible out-ofplane displacements ${ }^{10,11}$. Wrinkles were also observed in constrained annular dielectric elastomer actuators and the wavelength showed a dependence on the radius ratio of annular region ${ }^{12}$. Similarly, trumpet shaped elastomer membranes showed wrinkling behavior with the wavelength being influenced by the mechanical design and the prestretch of the membrane 
13. Greaney et al. proposed a theoretical model to describe both the onset of wrinkling as well as pull-in instability in dielectric elastomer membranes and made predictions that match the experimental results for wrinkling in circular and annular dielectric elastomer membranes ${ }^{14}$. Furthermore, wrinkling instabilities were observed in inflated dielectric elastomer balloons recently ${ }^{15-17}$.

It is significant to investigate mechanical and physical properties of dielectric elastomers, due to their extensive applications in stretchable electronics ${ }^{18}$, soft robots ${ }^{19,20}$, biomedical devices ${ }^{21}$, and energy harvesters ${ }^{22}$. In this paper, we observe various instability modes in a clamped circular membrane of a dielectric elastomer. Besides buckling and wrinkling, we find a new instability mode in dielectric elastomers - crumpling with focusing of stress on the surfaces of the dielectric elastomer. The radial prestretch is found to play a significant role in determining the instability mode of the membrane. The experiments show that the membrane may undergo buckling, wrinkling, or crumpling, when the prestretch is small, intermediate, or large, respectively. The finite element simulations agree with the experimental observations.

\section{Experimental results}

A membrane of dielectric elastomer, without or with the radial prestretch, is fixed onto a rigid circular frame (Fig. $1 \mathrm{a}-\mathrm{c}$ ). The diameter of the membrane is equal to the inner diameter (i.e., $6 \mathrm{~cm}$ ) of the rigid frame, which is aligned vertically. VHB 4910, 3M (with a thickness $H=$ $1 \mathrm{~mm}$ ) is employed as the elastomer. Carbon grease is coated on both the surfaces of the membrane to act as compliant electrodes and is connected to voltage (Fig. 1d). Voltage is programmed through an in-house custom code, and amplified by a high voltage amplifier (FR30P10, Glassman). We increase voltage at a small ramp rate of $20 \mathrm{~V} / \mathrm{s}$ to minimize the viscoelastic effects of the elastomer. Two cameras are employed to capture the front and top views of the membrane. 
The prestretch $\lambda_{\text {pre }}$ is varied from $\left(\lambda_{\text {pre }}=1,2, \ldots 6\right)$ to test the behaviour of the actuator at different prestretch levels. When the radial prestretch $\lambda_{\text {pre }}=1$, the membrane undergoes buckling. Buckling is the instability mode in which a membrane exhibits finite out of plane deformations with smooth deformation contours. As illustrated in Fig. 2a, a buckled membrane exhibits finite out-of-plane displacements and smooth deformation. When $\lambda_{\text {pre }}=$ 5 , the membrane undergoes wrinkling. Wrinkling is defined as the local out of plane deformations observed in a membrane upon loss of tension, with smooth stress contours often exhibiting wave like morphologies. Fig. 2b illustrates a wrinkled membrane with smooth deformation and negligible out-of-plane displacements. When $\lambda_{\text {pre }}=6$, the membrane undergoes crumpling which is defined as the instability with sharp singularities such as cones and ridges with stress concentrations around these singularities. As illustrated in Fig. 2c, a crumpled membrane exhibits finite out-of-plane displacements, but develops sharp singularities on its surfaces, which differentiates a crumpling mode from a buckling one. Besides the above observations, the membrane of $\lambda_{\text {pre }}=2,3$ or 4 may suffer dielectric breakdown without undergoing any instabilities. Table 1 summarizes all the experimental observations for the membrane of various radial prestretches. We first discuss the three instability modes- buckling, wrinkling, and crumpling, as observed in the experiments.

\subsection{Buckling}

When $\lambda_{\text {pre }}=1$, a dielectric elastomer buckles globally without forming any wrinkles locally on the surfaces. Movie 1 shows the electromechanical behavior of the membrane of $\lambda_{\text {pre }}=1$ undergoing buckling. When the voltage is small, the deformation of the membrane is negligible. When the voltage reaches $10 \mathrm{kV}$, the first-order buckling is triggered, and the membrane exhibits finite out-of-plane displacements. When the voltage reaches $22 \mathrm{kV}$, the membrane progresses into the second buckling mode. Fig. 3a shows the oblique and top views of the flat membrane at $\Phi=0$. Fig. $3 \mathrm{~b}$ shows the membrane at $\Phi=16 \mathrm{kV}$, when it has undergone the first-order buckling. The membrane bulges with axisymmetric deformation, exhibiting finite out-of-plane displacements, with an amplitude of $0.5 \mathrm{~cm}$. Fig. $3 \mathrm{c}$ shows the 
membrane at $\Phi=26 \mathrm{kV}$, when it has undergone the second-order buckling. As we can see, the boundary part (close to the rigid frame) forms a wavy pattern, which breaks the axisymmetric deformation of the entire membrane. These two buckling modes (Figs. $3 \mathrm{~b}$ and $3 \mathrm{c}$ ) are consistent with those simulated by finite element method which will be illustrated later. Fig. $3 \mathrm{~d}$ plots the buckling amplitude as a function of the voltage. It is found that the amplitudevoltage curve of the second-order buckling has a larger slope than that of the first-order buckling. This experimental observation is also qualitatively consistent with the simulations discussed in Section 3.1.

Voltage-induced buckling, as shown in Movie 1 and Fig. 3, can be interpreted as follows. When a dielectric elastomer of $\lambda_{\text {pre }}=1$ is connected to a power source, voltage induces the equal-biaxial Maxwell stresses onto the membrane ${ }^{23}$, and the membrane attempts to expand. Constrained by a rigid frame, the membrane is thus subject to the equal-biaxial compressive stresses. When the compressive stresses reach a critical value, the membrane buckles exhibiting finite out-of-plane displacements.

\subsection{Wrinkling}

The membrane of $\lambda_{\text {pre }}=5$ undergoes wrinkling when the voltage exceeds a critical value. Movie 2 shows the experimental behavior of a membrane of $\lambda_{\text {pre }}=5$, after onset of loss of tension. Before the onset of loss of tension, the membrane experiences mechanical tension due to the initial prestretch. As the voltage increases, the mechanical stresses decrease. At a critical voltage $\Phi=5.96 \mathrm{kV}$, the membrane undergoes loss of tension and wrinkles are initiated at the boundary of the membrane. The wrinkles propagate across the other regions until the entire membrane wrinkles displaying a hierarchical pattern. The membrane exhibits a continuous transition from the flat to wrinkled state, without undergoing electromechanical instability ${ }^{11}$. Fig. 4 shows the fully wrinkled membrane at $\Phi=5.96 \mathrm{kV}$. The image shows a pattern with wrinkles originating from several nodes distributed across the membrane.

\subsection{Crumpling}


The membrane of $\lambda_{\text {pre }}=6$ undergoes crumpling when the voltage exceeds a critical value. Movie 3 shows the electromechanical behavior of this membrane. When the voltage reaches $5.92 \mathrm{kV}$, the membrane starts to bulge out and exhibits finite out-of-plane displacements. Meanwhile, vertices and ridges appear on the membrane.

Fig. 5 shows the front view of the membrane at $\Phi=6.10 \mathrm{kV}$. The membrane consists of two characteristic features in a crumpled structure: singular vertices and curved ridges ${ }^{24}$. Although the words "buckle" and "crumple" are interchangeable in colloquial usage, following King et al. (2012), we use them in their technical sense to mean a sinusoidal, smooth deformation and a stress-focusing deformation, respectively. While a buckled membrane has a continuous stress field ${ }^{25}$, a crumpled membrane exhibits focusing of stress ${ }^{3}$. The simulation results in Section 3.3 will show that the membrane exhibits focusing of stress around these vertices and ridges. The morphology of a crumbed membrane is usually unsmooth and thus highly unpredictable. How to accurately quantify its amplitude deserves further studies.

Crumpling in a dielectric elastomer can be interpreted as follows. The membrane of $\lambda_{\text {pre }}=6$ is subject to equal-biaxial tensile stresses at $\Phi=0$. When voltage is small, there is no voltage-induced deformation, but the tensile stresses in the membrane decrease as the voltage increases. At a certain level of voltage, the membrane suffers loss of tension. When the voltage further increases, the membrane is subject to equal-biaxial compressive stresses. As we know, the membrane of a very large radial prestretch can actually be very stiff due to the strainstiffening effect. Thus, the membrane has a large bending rigidity, so that the membrane will bulge with a large wavelength and exhibit finite out-of-plane displacements, when the compressive stresses reach a critical value. Meanwhile, due to its small thickness (since $h=$ $\left.H \lambda_{\text {pre }}^{-2}\right)$, the membrane is subject to a large electric field $(E=\Phi / h)$, which generates large voltage-induced stresses onto the membrane. After loss of tension, the voltage also generates large compressive stresses which cause the membrane to crumple, undergoing large local deformations with focusing of stress. 
The phenomenon that a large stress can lead to crumpling in a sheet can be observed in our daily lives. For example, one can conduct a simple experiment at home. Take a drinking straw and slightly compress it axially at the two ends with two fingers. One would see the straw buckle first, taking an arched shape. Compress it further by increasing the compressive force. Beyond a critical force, the straw crumples forming a ridge at one point along the length. The two halves to the left and right of the crumpled region will now be almost stress free. Large stress is only focused in the small region around the singularity. This phenomenon is often accompanied by plastic deformation due to the focusing of stress along the ridges ${ }^{24}$. It is understood that beyond a critical compressive force, the crumpled state with large local deformations and largely stress free areas is energetically favorable to a buckled state with a smooth stress distribution ${ }^{24}$.

The experiments conclude that dielectric elastomers may undergo different instabilities (such as buckling, wrinkling, and crumpling), depending upon the prestretches of the membrane. However, the membrane of $\lambda_{\text {pre }}=2,3$ or 4 suffers dielectric breakdown without undergoing any instabilities. The reasons are as follows. The failure of dielectric breakdown can be statistically distributed in the experiments, and it might especially occur first at weak parts in the material due to impurities, flaws, cracks, etc. The process of sample preparation might also induce these weak points, which decreases the dielectric strength of material and makes the membrane suffer dielectric breakdown before undergoing any instabilities. In the next section, we discuss finite element analysis to interpret all the experimental observations including buckling, wrinkling and crumpling.

\section{Finite element analysis using ABAQUS}

We model the elastomers based on the nonlinear field theory of dielectric elastomers in ABAQUS using a user-subroutine (UMAT) ${ }^{26}$. Since the prestretches vary across a large range, we employ the Gent model to capture the strain stiffening effect ${ }^{27}$. For more 
information about the method and code implementation, please refer to Foo and Zhang (2015) ${ }^{28}$. We simulate the membrane of a dielectric elastomer (Fig. 1) using the static analysis in Abaqus/Standard, since a low voltage ramp rate of $20 \mathrm{~V} / \mathrm{s}$ is employed in the experiments. We adopt an elastic model with the material parameters (i.e., $\mu=43 \mathrm{kPa}, J_{l i m}=115$, and $\varepsilon=$ $4.12 \times 10^{-11} \mathrm{~F} / \mathrm{m}^{11}$ ) and configuration parameters (i.e., $H=1 \mathrm{~mm}$, and $R \lambda_{\text {pre }}=3 \mathrm{~cm}$ ). Similar to Henann et al. (2013) ${ }^{29}$, we neglect the fringe effects of the electric field and the failure due to dielectric breakdown. The simulations show that dielectric elastomers of various radial prestretches may undergo buckling, wrinkling, and crumpling.

\section{1. Buckling}

Movie 4 shows the simulation results of the membrane of $\lambda_{p r e}=1$. As we can see, when the voltage reaches a critical value $\Phi=1.5 \mathrm{kV}$, the membrane buckles, exhibiting finite out-ofplane displacements. When the voltage reaches another critical value $\Phi=9.9 \mathrm{kV}$, the membrane undergoes the second-order buckling.

Fig. 6a shows the flat membrane at $\Phi=0$, and two sample points are marked on the membrane. Point $A$ is in the center of the membrane, while Point $B$ is close to the fixed boundary. Fig. $6 \mathrm{~b}$ shows the front and top views of the membrane at $\Phi=2 \mathrm{kV}$. The entire membrane bulges, when undergoing the first-order buckling. Fig. $6 \mathrm{c}$ shows the membrane at $\Phi=20 \mathrm{kV}$. The boundary part (containing Point $B$ ) forms a wavy pattern in the circumferential direction when the membrane undergoes the second-order buckling. Fig. 9d shows the out-ofplane displacement as a function of the voltage, for Point $A$ or $B$. As shown in Fig. 6d, at $\Phi=$ $1.5 \mathrm{kV}$, Points $A$ and $B$ initiate out-of-plane displacements, representing the onset of the firstorder buckling. The buckling of the membrane may result from inherent numerical perturbations during the computations, due to geometric imperfections (say, irregular element geometries). In addition, since the membrane is vertically placed in the experiments, we consider the gravity of material in the computations, which may lead to inhomogeneous thickness in the membrane. The non-uniform thickness may also function as disturbance for 
buckling/instability. At $\Phi=9.9 \mathrm{kV}$, the second-order buckling is triggered. As shown in Movie 4 and Fig. 6c, during the second-order buckling, the center of membrane still bulges away from the initial plane of the membrane, however, the part close to the boundary forms a wavy pattern in the circumferential direction, which breaks the axisymmetric deformation of the membrane. Consequently, as shown in Fig. 6d, although the out-of-plane displacement of Point $A$ increases, the displacement of Point $B$ decreases at the initiation of the second-order buckling.

Comparing Figs. $3 \mathrm{~b}$ to $6 \mathrm{~b}$, and Figs. $3 \mathrm{c}$ to $6 \mathrm{c}$, the simulation results of the first and second-order buckling modes are qualitatively consistent with the experimental observations. However, there is a large discrepancy in the critical voltage for the first-order or second-order buckling. The main reason is that an elastic material model is employed in the finite element simulations, while the elastomer - VHB is well known for its high viscoelasticity ${ }^{30}$. How to model and analyze buckling or other instabilities in a viscoelastic dielectric elastomer deserves further investigation.

\subsection{Wrinkling}

Movie 5 shows the simulation results of the membrane of $\lambda_{\text {pre }}=5$. As we can see, when the voltage reaches a critical value $\Phi=8.72 \mathrm{kV}$, wrinkles start to nucleate in the membrane. Then the wrinkles quickly propagate throughout the area between $8.72 \mathrm{kV}$ and $8.78 \mathrm{kV}$. Fig. $7 \mathrm{a}$ shows the simulation results for the wrinkled membrane, which is qualitatively similar to the experimental observation as shown in Fig. 4. Fig. 7b shows the distribution of the hoop stress $\left(\sigma_{\theta}\right)$. As we can see, the stresses are distributed smoothly throughout the membrane, which makes a wrinkled membrane different from a crumpled one (as will be discussed in the next subsection). It should also be noted that the membrane of $\lambda_{\text {pre }}=5$, unlike a buckled membrane of $\lambda_{\text {pre }}=1$, exhibits only local out of plane deformations corresponding to the crests and troughs of the wrinkles, while globally remaining within the same plane. 
The simulations also show the membrane of $\lambda_{\text {pre }}=2,3$, or 4 undergoes the similar instability of wrinkling, when the voltage reaches a certain level. However, these membranes suffer dielectric breakdown in the experiments, without exhibiting any instabilities. The reasons for the discrepancy between the simulations and the experiments are as follows. In the simulations, the failure of dielectric breakdown is not taken into account, and the membrane undergoes wrinkling when the voltage is large enough to induce loss of tension of the membrane. However, in the experiments dielectric breakdown may occur before initiation of wrinkling, so that instability of wrinkling is not observed for the membrane of $\lambda_{\text {pre }}=2,3$, or 4.

\subsection{Crumpling}

Movie 6 shows the simulation results of the membrane of $\lambda_{\text {pre }}=6$. When the voltage reaches a critical value ( $\Phi=8.35 \mathrm{kV})$, the membrane is subject to the critical equal-biaxial compressive stresses which make the membrane bulge, exhibiting finite out-of-plane displacements. Meanwhile, the membrane undergoes crumpling, consisting of singular vertices and long curved ridges on the surfaces.

Fig. 8a shows the pattern of the crumpled membrane, in which we can observe singular vertices connected by curved ridges. Fig. $8 \mathrm{~b}$ shows the distribution of the hoop stress $\left(\sigma_{\theta}\right)$. As we can see, the membrane exhibits focusing of stress along the ridges (in red), which are much larger than those in other areas (in green). These simulations are qualitatively consistent with the experimental observations, in which out-of-plane deformation with singular vertices and curved ridges has been observed (Fig. 5).

Comparing Fig. 7 (for the wrinkled membrane) to Fig. 8 (for the crumpled membrane), we can find that in these two different states, their stress distributions are quite different. The in-plane stress of a wrinkled membrane is dominated by negative values, indicating the membrane is under compression. As we can also see, the distribution of stress of the wrinkled membrane is more uniform and smoother, compared to that of a crumpled membrane. The 
in-plane stress of a crumpled membrane shows localizations at the ridges, where the geometrical singularities are formed and high stress concentrations are developed.

The simulations show that a dielectric elastomer may undergo buckling, wrinkling, or crumpling, respectively, well consistent with the experiments. In the next section, we interpret the effect of prestretch on the instability mode through a theoretical analysis of the parameters on the mechanical properties of the membrane.

\section{Discussions}

Both the experiments and simulations show that a dielectric elastomer may undergo different instability modes, depending on the applied radial prestretch $\lambda_{\text {pre }}$. The simulation results predict that a dielectric elastomer may undergo buckling when $\lambda_{\text {pre }}$ is small $\left(\lambda_{\text {pre }} \leq 2\right)$, undergo wrinkling when $\lambda_{\text {pre }}$ is intermediate $\left(2<\lambda_{\text {pre }} \leq 5.5\right)$ and undergo crumpling when $\lambda_{\text {pre }}$ is large $\left(\lambda_{\text {pre }}>5.5\right)$.

The effects of $\lambda_{\text {pre }}$ on instability modes can be interpreted as follows. Based on theory of linear elasticity, a membrane may respond to forces by stretching and bending, with respective moduli $M_{1}=E_{S} h$ and $M_{2}=E_{S} h^{3} /\left[12\left(1-v^{2}\right)\right]$, where $E_{S}$ is the Young's modulus, $h$ is the thickness, and $v$ is the Poisson ratio ${ }^{31}$. The different dependencies of $M_{1}$ and $M_{2}$ on $h$ explain why stretching/compression in a thin sheet is energetically costlier than bending. Consequently, to reduce its elastic energy, a thin sheet can easily buckle into shapes that remove in-plane compression. The smaller the bending rigidity or the larger the compression, the smaller the wavelength of the buckles/wrinkles ${ }^{32}$.

The radial prestretch $\lambda_{\text {pre }}$ may affect the electromechanical behavior of a dielectric elastomer by affecting the bending rigidity in two aspects - stiffness and thickness. Fig. 9 plots the stress-stretch curve and the thickness-stretch curve, when the membrane is subject to equal-biaxial stresses/strains. In plotting Fig. 9, we use the same material model as before (i.e., 
the Gent material model with $\mu=43 \mathrm{kPa}$ and $\left.J_{\text {lim }}=115\right)$. The stress in Fig. 9 can be the total stress consisting of both the mechanical stress and the Maxwell stress (due to the applied voltage). We define the stiffness as the increase in the total stress required to cause an infinitesimal increase in the stretch, i.e., the slope of the stress-stretch curve in Fig. 9. We assume that the membrane is flat at the onset of instability. The instability mode and the corresponding wavelength depend on the stiffness and the thickness of the membrane at the flat state ${ }^{1}$.

When $\lambda_{\text {pre }}$ is small $\left(\lambda_{\text {pre }} \leq 2\right)$, the slope of the stress-stretch curve is relatively large, which represents a large stiffness. Meanwhile, as illustrated in Fig. 9, the thickness of the membrane is large. As a result, its bending rigidity is large, so that the wavelength of the buckles is also large. In addition, the amplitude of the buckles/wrinkles generally has a similar length scale as that of the wavelength ${ }^{1}$. Consequently, the membrane of a small $\lambda_{\text {pre }}$ undergoes buckling, exhibiting a large wavelength and finite out-of-plane displacements. The instability modes for the membranes of various prestretches, predicted by the finite element simulations, are also illustrated in Fig. 9.

When $\lambda_{\text {pre }}$ is intermediate $\left(2<\lambda_{\text {pre }} \leq 5.5\right)$, the slope of the stress-stretch curve is small, which represents a small stiffness. Meanwhile, the thickness of the membrane is also small since $h=H \lambda_{\text {pre }}^{-2}$, where $H$ is the thickness at the reference state and $h$ is the thickness at the prestretched state. As a result, its bending rigidity is small, and the wavelength of the wrinkles is small. The amplitude of the wrinkles is proportional to the wavelength of the wrinkles and is also small, so that the membrane exhibits negligible out-of-plane displacements.

The membrane of a large radial prestretch $\left(\lambda_{\text {pre }}>5.5\right)$ is expected to undergo crumpling. This prediction can be understood as follows. Subject to a large $\lambda_{\text {pre }}$, the membrane is very stiff due to the strain-stiffening effect. However, since the membrane is completely constrained by the rigid frame, its thickness may not decrease before the onset of 
instability. As a result, the membrane may have a large bending rigidity due to the dominant effect of high stiffness. When the voltage reaches a critical value, the membrane may bulge, exhibiting a large wavelength and finite out-of-plane displacements. On the other hand, compared to a buckled membrane of small $\lambda_{\text {pre }}$, at the same range of voltage, the membrane of very large $\lambda_{\text {pre }}$ is subject to the Maxwell's stresses which can be two orders higher, due to its much smaller thickness. Owing to this observation, very large compressive stresses are generated in the latter after loss of tension. As discussed in Section 2.3, large compressive stresses can cause a sheet to crumple 24,33 .

Besides the prestretch, mechanical design parameters, such as the ratio of active area in the membrane to the passive area, may also affect electromechanical behavior of a dielectric elastomer. We studied the instability modes in a circular dielectric elastomer membrane with a large passive area (see figure 1 of [16]). This membrane is partially coated with compliant electrodes in the central circular region, while the passive part of the membrane is fixed to a rigid frame. Both the experiments and the finite element simulations showed the membrane did not undergo crumpling (especially at a large prestretch), but exhibited wrinkling. The reasons are as follows. For a dielectric elastomer with a large passive region, the active region expands when subject to voltage. The membrane may decrease its thickness and its bending rigidity. Consequently, the membrane of a large radial prestretch undergoes wrinkling rather than crumpling. In this paper, both the experiments and the simulations show that the crumpling instability can occur at strict design conditions: a very large radial prestretch $\left(\lambda_{\text {pre }}>5.5\right)$ and a completely constrained membrane. It should be noted that a crumpled membrane of dielectric elastomer, with finite out of plane deformation and focusing of stress, is not frequently observed in the experiments and thus is not well studied in literature, due to these strict design conditions.

\section{Concluding remarks}


This paper investigates voltage-induced instabilities in a circular membrane of a dielectric elastomer fixed to a rigid frame. The experiments show that the membrane may undergo buckling, wrinkling, or crumpling, when the voltage reaches a certain level. The radial prestretch plays a significant role in determining its instability mode. For example, when the prestretch is small, the membrane may undergo buckling. When the prestretch is intermediate, the membrane may undergo wrinkling. Besides buckling and wrinkling, we also observe a new instability mode - crumpling with focusing of stress on the surfaces of the membrane. Crumpling in a dielectric elastomer may occur at a very large prestretch in a fully constrained membrane. These loading conditions lead to a large bending rigidity of the membrane and large compressive stresses upon loss of tension causing the membrane to crumple. Due to these strict conditions, crumpling in a dielectric elastomer is not frequently observed in the experiments, and thus is not well investigated in literature. Finite element simulations are conducted to study the electromechanical behavior of buckling, wrinkling or crumpling. The simulation results are well consistent with the experimental observations. We wish that investigation of mechanical and physical properties of dielectric elastomers can enhance their extensive significant applications to soft devices and soft robots.

\section{Acknowledgements}

J.Z. thanks MOE Tier 1, Singapore (grant number R-265-000-609-114) and A*STAR, Singapore (grant number R-265-000-629-305). 


\section{References}

1 E. Cerda and L. Mahadevan, Phys. Rev. Lett., 2003, 90, 74302.

2 Y. Klein, E. Efrati and E. Sharon, Science (8o)., 2007, 315, 1116-1120.

3 H. King, R. D. Schroll, B. Davidovitch and N. Menon, Proc. Natl. Acad. Sci., 2012, 109, 9716-9720.

$4 \quad$ R. Pelrine, R. Kornbluh, Q. Pei and J. Joseph, Science (8o)., 2000, 287, 836-839.

$5 \quad$ S. Ashley, Sci. Am., 2003, 289, 52-59.

6 F. Carpi, S. Bauer and D. De Rossi, Science (8o)., 2010, 330, 1759-1761.

S. Rosset, M. Niklaus, P. Dubois and H. Shea, Sensors Actuators A, 2008, 144, 185193.

8 B. Tavakol, M. Bozlar, C. Punckt, G. Froehlicher, H. A. Stone, I. A. Aksay and D. P. Holmes, Soft Matter, 2014, 10, 4789-4794.

9 H. Bense, M. Trejo, E. Reyssat, J. Bico and B. Roman, Soft Matter, 2017, 13, 28762885 .

10

11

12

13

14

15

16

17

18

19

J.-S. Plante and S. Dubowsky, Int. J. Solids Struct., 2006, 43, 7727-7751.

H. Godaba, Z.-Q. Zhang, U. Gupta, C. Chiang Foo and J. Zhu, Soft Matter, 2017, 13, 2942-2951.

K. Li, W. Wu, Z. Jiang and S. Cai, J. Appl. Mech., 2018, 85, 11007.

G. Mao, L. Wu, Y. Fu, J. Liu and S. Qu, AIP Adv., 2018, 8, 35314.

P. Greaney, M. Meere and G. Zurlo, J. Mech. Phys. Solids, 2019, 122, 84-97.

T. Li, C. Keplinger, R. Baumgartner, S. Bauer, W. Yang and Z. Suo, J. Mech. Phys. Solids, $2013,61,611-628$.

G. Mao, X. Huang, M. Diab, T. Li, S. Qu and W. Yang, Soft Matter, 2015, 11, 6569-6575.

G. Mao, Y. Xiang, X. Huang, W. Hong, T. Lu and S. Qu, J. Appl. Mech., 2018, 85, 71003.

C. Keplinger, J.-Y. Sun, C. C. Foo, P. Rothemund, G. M. Whitesides and Z. Suo, Science, 2013, 341, 984-7.

Y. Wang and J. Zhu, Extrem. Mech. Lett., 2016, 6, 88-95. 
H. Godaba, J. Li, Y. Wang and J. Zhu, IEEE Robot. Autom. Lett., 2016, 1, 624-631.

21 L. G. Ledgerwood, S. Tinling, C. Senders, A. Wong-Foy, H. Prahlad and T. T. Tollefson, Arch. Facial Plast. Surg., 2012, 14, 413-8.

R. Kaltseis, C. Keplinger, S. J. Adrian Koh, R. Baumgartner, Y. F. Goh, W. H. Ng, A. Kogler, A. Tröls, C. C. Foo, Z. Suo and S. Bauer, RSC Adv., 2014, 4, 27905-27913. Z. Suo, Acta Mech. Solida Sin., 2010, 23, 549-578.

M. Ben Amar and Y. Pomeau, Proc. R. Soc. A Math. Phys. Eng. Sci., 1997, 453, 729755 .

25 P. S. Bulson, The stability of flat plates, Elsevier Publishing Company, 1969.

26 Z. Suo, X. Zhao and W. H. Greene, J. Mech. Phys. Solids, 2008, 56, 467-486.

27 A. N. Gent, Rubber Chem. Technol., 1996, 69, 59-61.

28 C. C. Foo and Z.-Q. Zhang, Int. J. Appl. Mech., 2015, 7, 1550069.

29 D. L. Henann, S. A. Chester and K. Bertoldi, J. Mech. Phys. Solids, 2013, 61, 20472066.

30

M. Kollosche, G. Kofod, Z. Suo and J. Zhu, J. Mech. Phys. Solids, 2015, 76, 47-64.

31

J. W. S. Rayleigh, The theory of sound, Dover, New York, 1945.

32

E. Sharon, B. Roman, M. Marder, G.-S. Shin and H. L. Swinney, Nature, 2002, 419, 579.

33

A. Lobkovsky, S. Gentges, H. Li, D. Morse and T. A. Witten, Science (8o)., 1995, 270, $1482-1485$. 


\section{Figures}

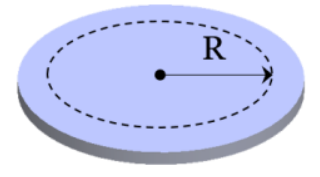

(a) Reference state

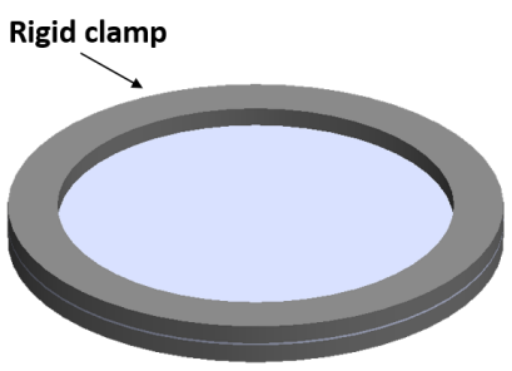

(c) Prestretched and clamped state

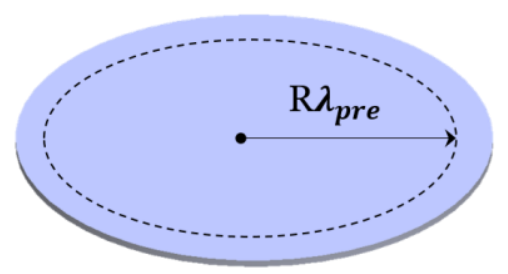

(b) Prestretched state

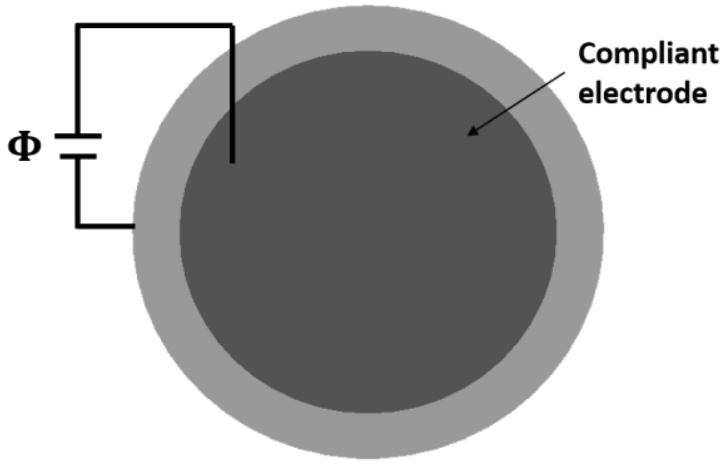

(d)Actuated state

Fig. 1 Schematic of a membrane of a dielectric elastomer. (a-b) A circular membrane of radius, $R$ is prestretched to a radius, $\mathrm{R} \lambda_{\text {pre }}$. (c) The prestretched membrane is clamped using rigid annular frames. (d) The membrane is coated with compliant electrodes and subjected to a voltage $\Phi$. 


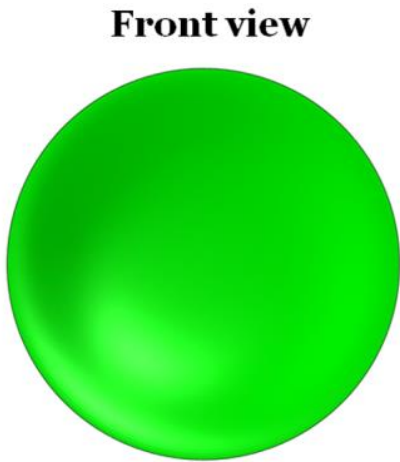

Top view

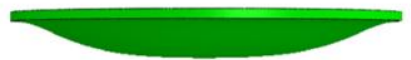

(a)

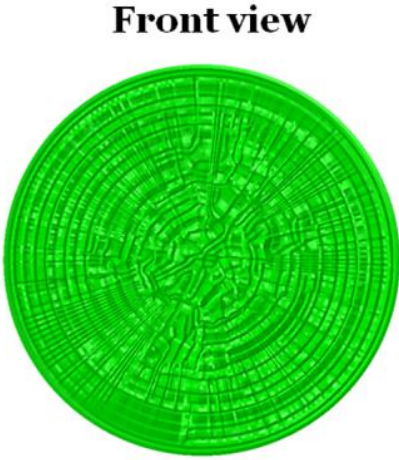

Top view

(b)

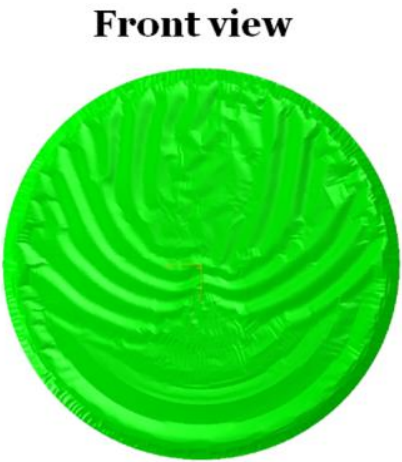

Top view

(c)

Fig. 2 Schematics of instability modes of buckling, wrinkling, and crumpling. Front and top views of a buckled membrane (a), a wrinkled membrane (b), and a crumpled membrane (c). 


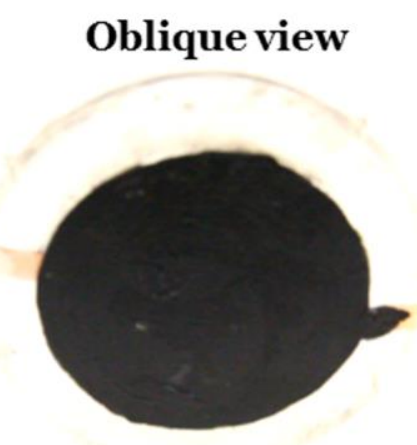

Top view

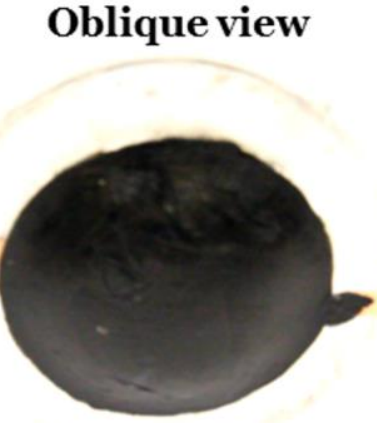

Top view
Oblique view

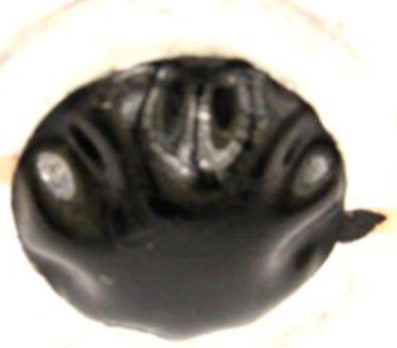

Top view

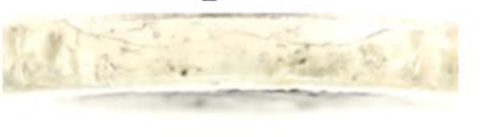

(a) $\Phi=0 \mathrm{kV}$

(b) $\Phi=16 \mathrm{kV}$

(c) $\Phi=26 \mathrm{kV}$

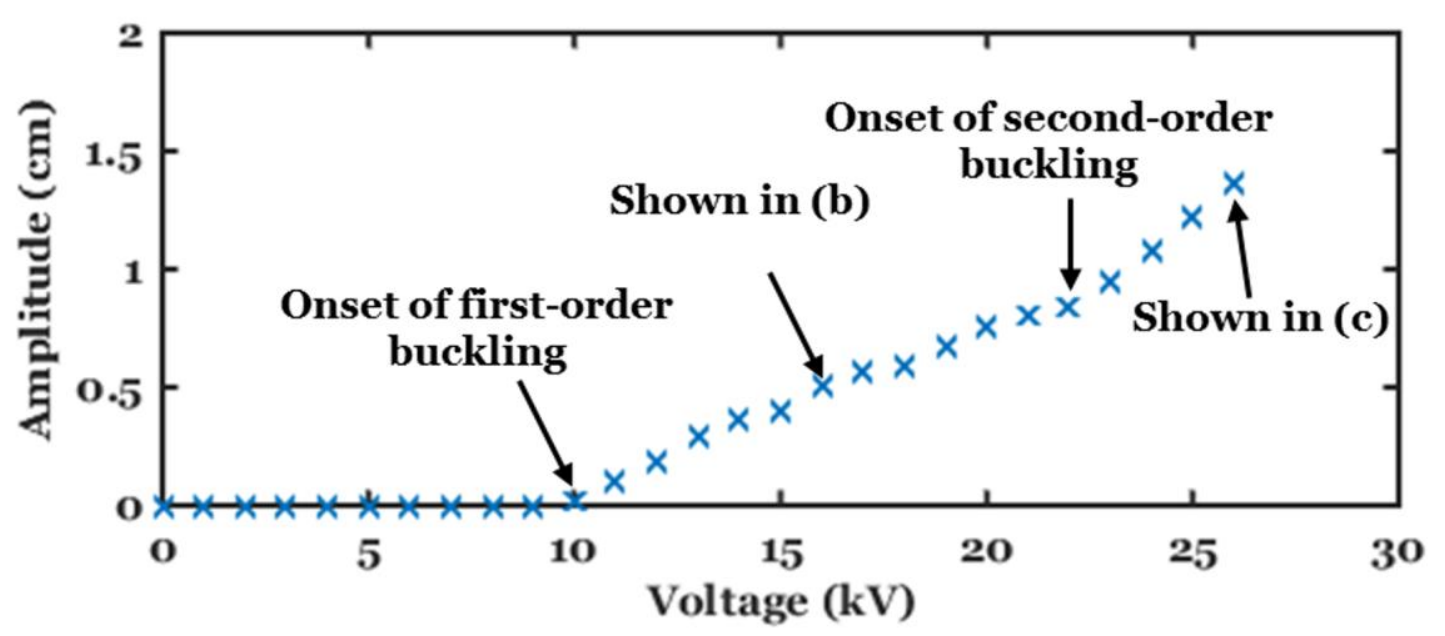

(d) Amplitude of buckling versus applied voltage

Fig. 3 Buckling of the membrane of $\lambda_{\text {pre }}=1$. (a) Oblique and top views of the flat membrane at $\Phi$ $=0$. (b) The membrane at $\Phi=16 \mathrm{kV}$. The membrane undergoes the first-order buckling. (c) The membrane at $\Phi=26 \mathrm{kV}$. The membrane undergoes the second-order buckling. (d) Buckling amplitude as a function of voltage. The first or second-order buckling is triggered at $\Phi=10 \mathrm{kV}$, or $22 \mathrm{kV}$, respectively. 


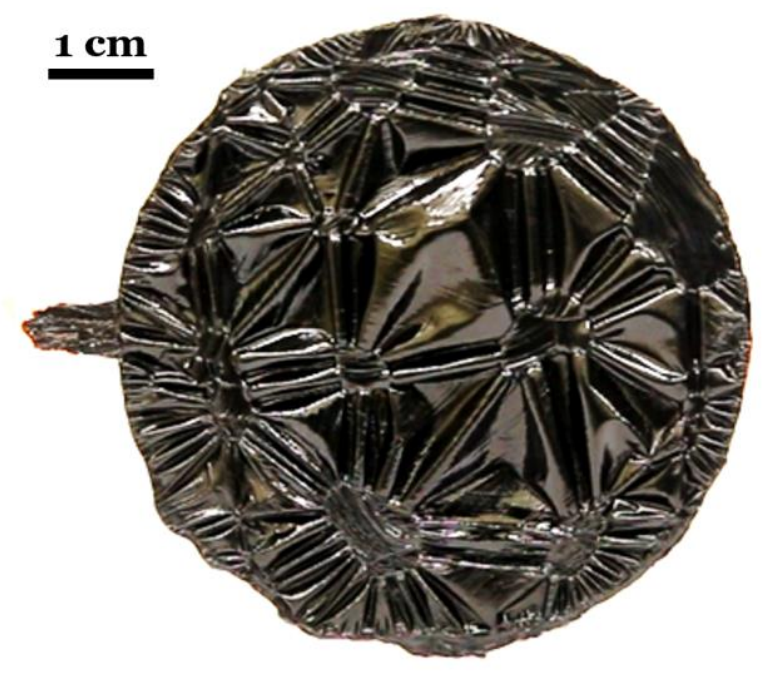

Fig. 4 Hierarchical wrinkling pattern in a membrane of $\lambda_{\text {pre }}=5$ at $\Phi=5.96 \mathrm{kV}$ 


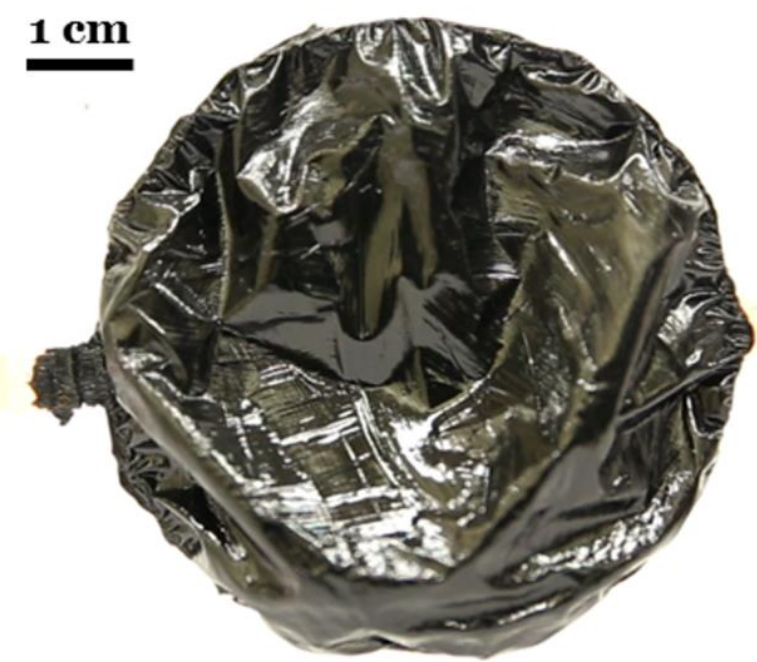

Fig. 5 The membrane of $\lambda_{\text {pre }}=6$ undergoes crumpling at $\Phi=6.10 \mathrm{kV}$. 


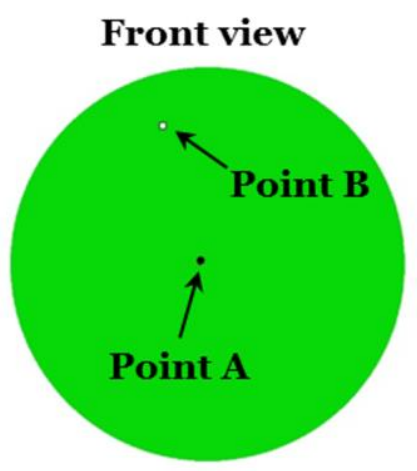

Top view

(a) $\Phi=0 \mathrm{kV}$

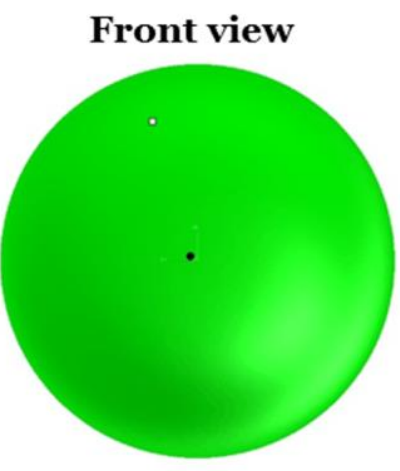

Top view

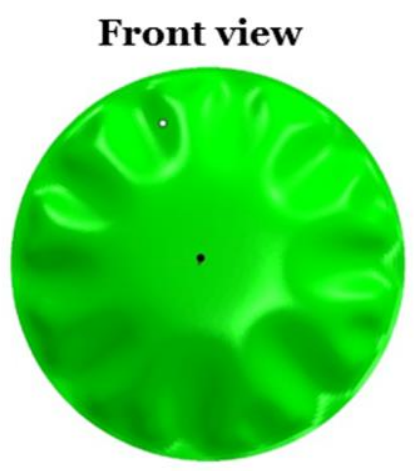

Top view

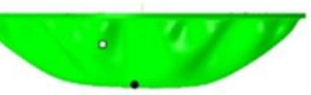

(c) $\Phi=\mathbf{2 0} \mathrm{kV}$

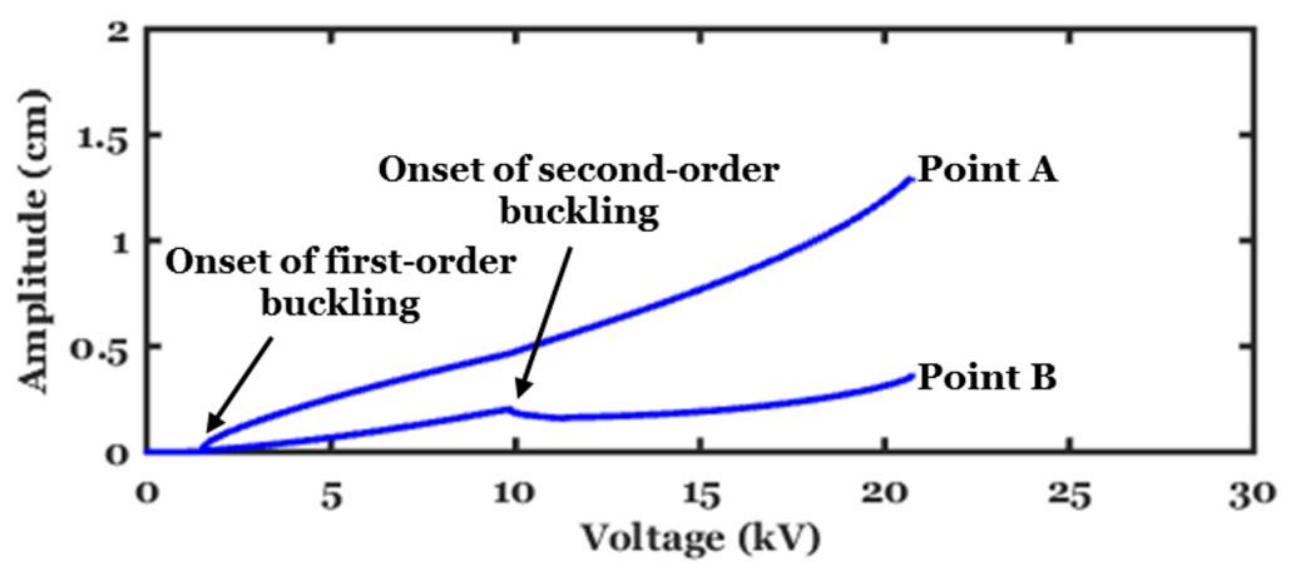

(d) Amplitude of buckling versus applied voltage

Fig. 6 Simulations of a buckled membrane. (a) Front and top views of the flat membrane at $\Phi=$ o. Points $A$ and $B$ are two sample points on the membrane. (b) The membrane at $\Phi=2 \mathrm{kV}$. The membrane undergoes the first-order buckling. (c) The membrane at $\Phi=20 \mathrm{kV}$. The membrane undergoes the second-order buckling. (d) Buckling amplitude as a function of voltage for Point $A$ or $B$. The first or second-order buckling is triggered at $\Phi=1.5 \mathrm{kV}$, or $9.9 \mathrm{kV}$, respectively. 


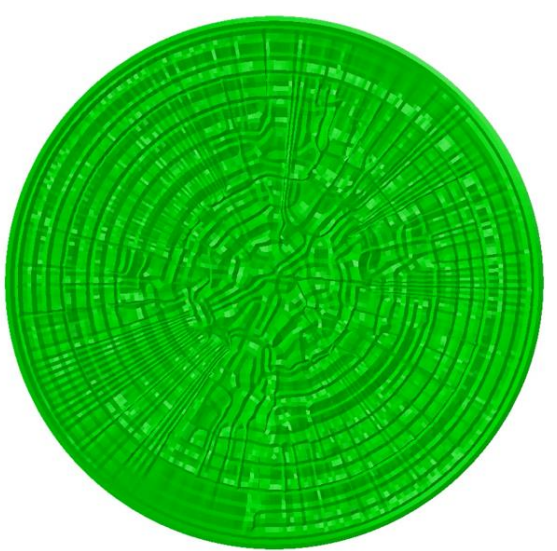

(a)

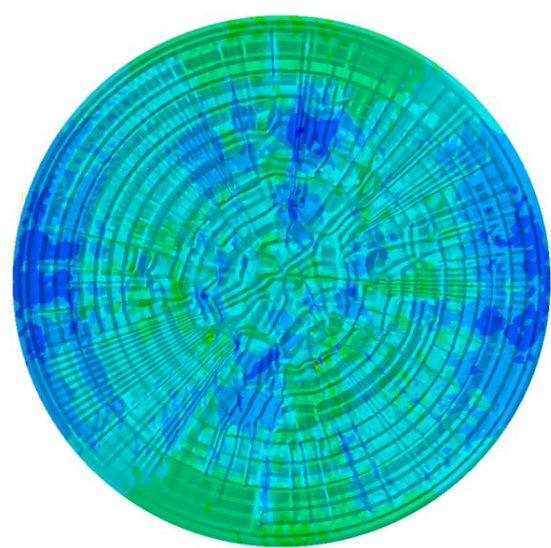

(b)

b)

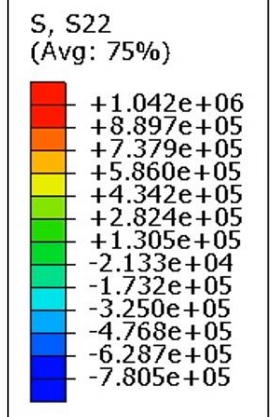

Fig. 7 Simulations of a wrinkled membrane. A membrane of $\lambda_{\text {pre }}=5$ undergoes wrinkling. 


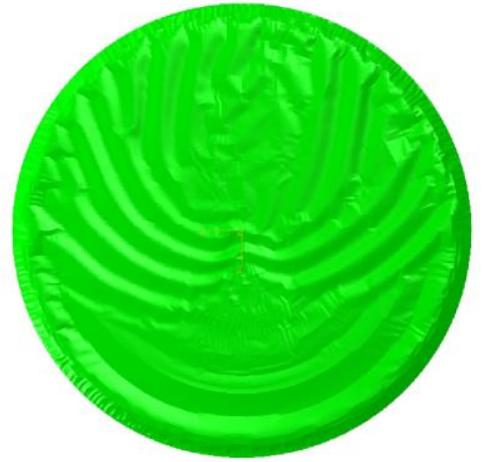

(a)

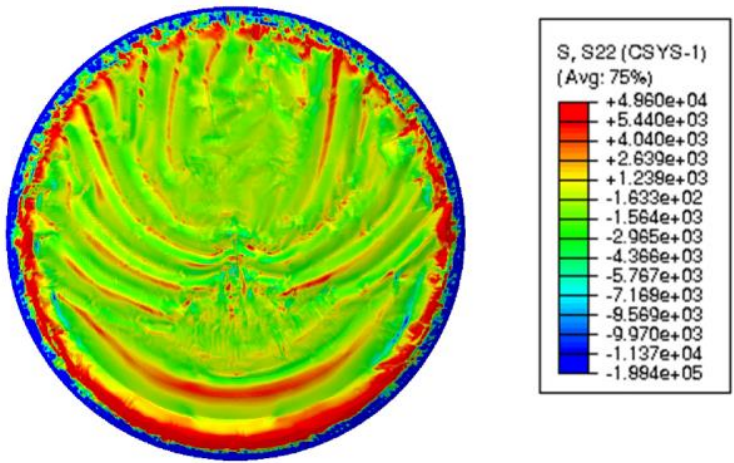

(b)

Fig. 8 Simulations of a crumpled membrane (a) The pattern of the membrane (b) The membrane with distribution of the hoop stress $\left(\sigma_{\theta}\right)$. The membrane exhibits focusing of stress around singular vertices and curved ridges that connect the vertices. 


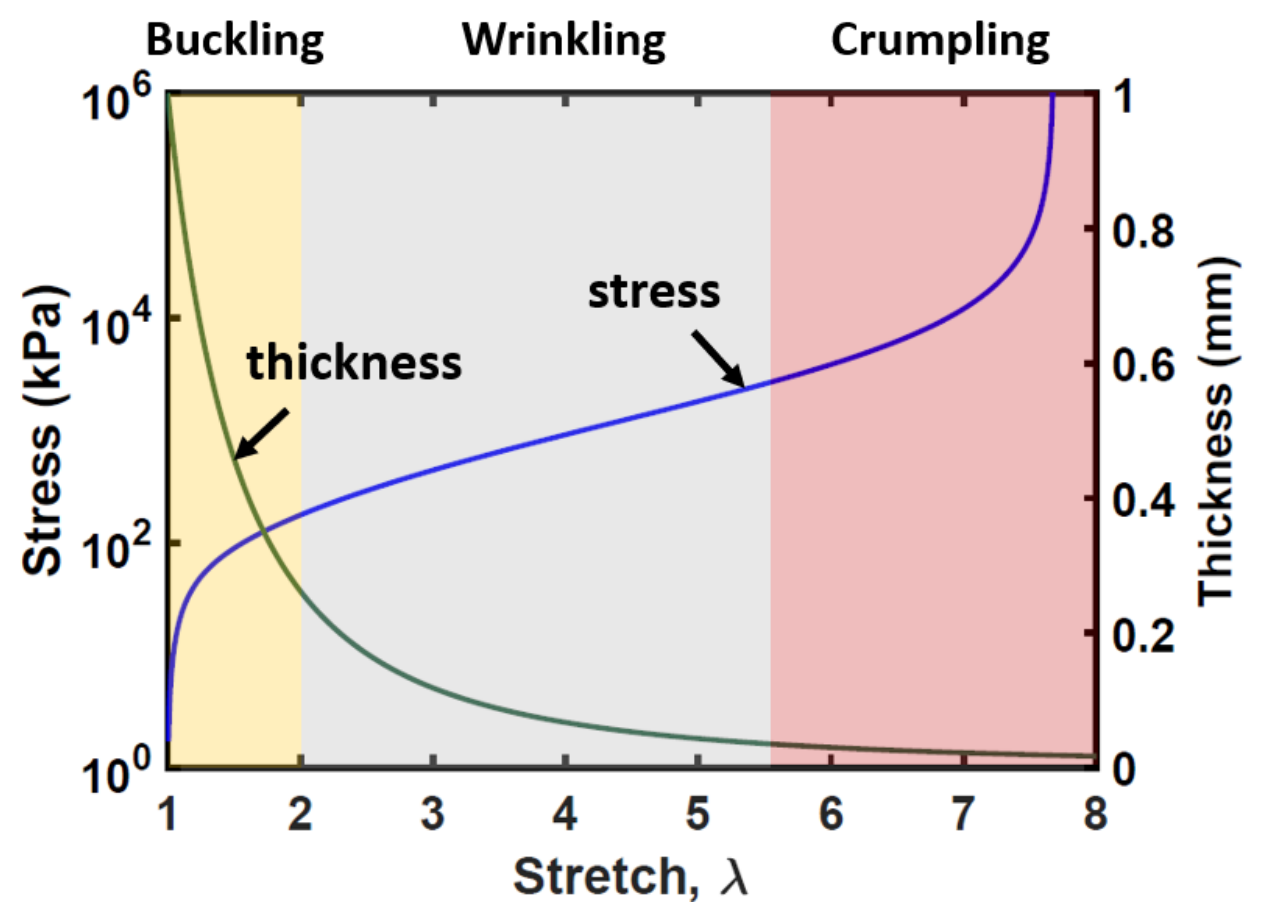

Fig. 9 Stress and thickness of the membrane as a function of stretch. Instability modes predicted by finite element results show buckling $\left(\lambda_{\text {pre }}<2\right)$, wrinkling $\left(2 \leq \lambda_{\text {pre }} \leq 5.5\right)$, and crumpling $\left(\lambda_{\text {pre }}>5.5\right)$. 
Table 1. Experimental observations of dielectric elastomers of various prestretches

\begin{tabular}{|l|l|l|}
\hline Prestretch & Experimental observation & Characteristics \\
\hline 1 & Buckling & $\begin{array}{l}\text { Out-of-plane deformation, } \\
\text { Smooth strain/stress }\end{array}$ \\
\hline 2 & Dielectric breakdown & Without any instabilities \\
\hline 3 & Dielectric breakdown & Without any instabilities \\
\hline 4 & Wrinkling & Without any instabilities \\
\hline 5 & Crumpling & $\begin{array}{l}\text { Without out-of-plane deformation, } \\
\text { Smooth strain/stress }\end{array}$ \\
\hline 6 & & $\begin{array}{l}\text { Out-of-plane deformation, } \\
\text { Focusing of stress }\end{array}$ \\
\hline
\end{tabular}


Instabilities in dielectric elastomers: buckling, wrinkling, and crumpling

Hareesh Godaba, Zhi-Qian Zhang, Ujjaval Gupta, Choon Chiang Foo, Jian Zhu

Dielectric elastomers undergo different modes of instabilities upon loss of tension- buckling, wrinkling and crumpling, depending on the loading conditions.

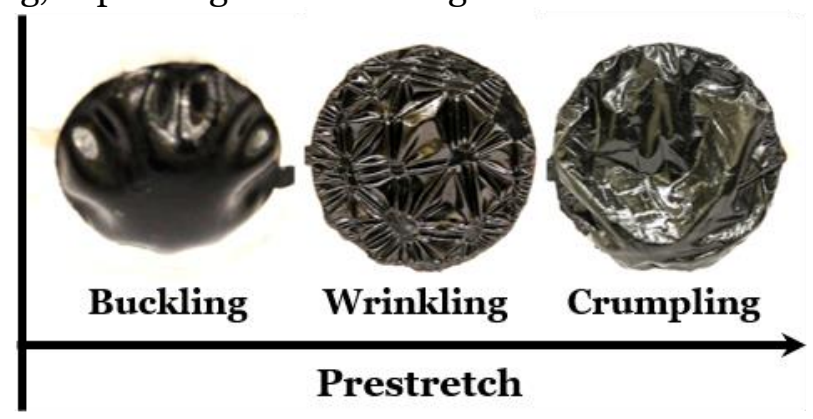

